\title{
An exploratory pilot study of mechanisms of action within normative feedback for adult drinkers
}

Alexis Kuerbis, Frederick J. Muench, Rufina Lee, Juan Pena, Lisa Hail

Background: Normative feedback (NF), or receiving information about one's drinking compared to peer drinking norms, is one of the most widely used brief interventions for prevention and intervention for hazardous alcohol use. NF has demonstrated predominantly small but significant effect sizes for intention to change and other drinking related outcomes. Identifying mechanisms of action may improve the effectiveness of NF; however, few studies have examined NF's mechanisms of action, particularly among adults. Objective: This study is an exploratory analysis of two theorized mechanisms of NF: discrepancy (specifically personal dissonance-the affective response to feedback) and belief in the accuracy of feedback. Method: Using Amazon's Mechanical Turk, 87 men $(n=56)$ and women $(n=31)$ completed an online survey during which they were asked about their perceptions about their drinking and actual drinking behaviors. Then participants were provided tailored NF and evaluated for their reactions. Severity of discrepancy was measured by the difference between one's estimated percentile ranking of drinking compared to peers and actual percentile ranking. Surprise and worry reported due to the discrepancy were proxies for personal dissonance. Participants were also asked if they believed the feedback and if they had any plans to change their drinking. Mediation analyses were implemented, exploring whether surprise, worry, or belief in the accuracy of feedback mediated severity of discrepancy's impact on plan for change. Results: Among this sample of adult drinkers, severity of discrepancy did not predict plan for change, and personal dissonance did not mediate severity of discrepancy. Severity of discrepancy was mediated by belief in the accuracy of feedback. In addition, viewing one's drinking as a problem prior to feedback and post-NF worry both predicted plan for change independently. Conclusions: Results revealed that NF may not work to create personal dissonance through discrepancy, but belief in the accuracy of feedback may be important. It appears the more one believes the feedback, the more one makes a plan for change, suggesting practitioners should be mindful of how information within feedback is presented. Findings also indicate NF may work by validating a preexisting perception that drinking is a problem instead of creating concern related to discrepancy where none existed. Limitations regarding generalizability are discussed. 
1 Running header: EXPLORING MECHANISMS OF FEEDBACK

2 Abstract word count: 360

3 Word count: 4,569

4 Tables: 4

5 Figures: 0

6 Number of references:

7 An exploratory pilot study of mechanisms of action within normative feedback for adult drinkers

8

\author{
Alexis N. Kuerbis, LCSW, Ph.D. ${ }^{1}$ \\ Frederick J. Muench, Ph.D. ${ }^{2}$ \\ Rufina Lee, Ph.D. ${ }^{1}$ \\ Juan Peña, Ph.D. ${ }^{1}$ \\ Lisa Hail, $\mathrm{MA}^{3}$
}

Affliations: ${ }^{1}$ Silberman School of Social Work, Hunter College at the City University of New York, New York, NY; ${ }^{2}$ Northwell Health, Great Neck, NY; ${ }^{3}$ Fairleigh Dickinson University, Teaneck, NJ

Author Note: This work was funded by National Institute on Alcohol Abuse and Alcoholism

Grant R34 AA 021502A (PI: Muench). Correspondence concerning this article should be addressed to Alexis Kuerbis, c/o Silberman School of Social Work, 2180 Third Avenue, Rm 435, New York, NY 10035. E-mail: ak1465@hunter.cuny.edu.

27 Declaration of Interest: The authors report no conflicts of interest. The authors alone are responsible for the content and writing of this paper 
31

\begin{abstract}
Background: Normative feedback (NF), or receiving information about one's drinking compared to peer drinking norms, is one of the most widely used brief interventions for prevention and intervention for hazardous alcohol use. NF has demonstrated predominantly small but significant effect sizes for intention to change and other drinking related outcomes. Identifying mechanisms of action may improve the effectiveness of NF; however, few studies have examined NF's mechanisms of action, particularly among adults.
\end{abstract}

Objective: This study is an exploratory analysis of two theorized mechanisms of NF: discrepancy (specifically personal dissonance - the affective response to feedback) and belief in the accuracy of feedback.

Method: Using Amazon's Mechanical Turk, 87 men $(n=56)$ and women $(n=31)$ completed an online survey during which they were asked about their perceptions about their drinking and actual drinking behaviors. Then participants were provided tailored NF and evaluated for their reactions. Severity of discrepancy was measured by the difference between one's estimated percentile ranking of drinking compared to peers and actual percentile ranking. Surprise and worry reported due to the discrepancy were proxies for personal dissonance. Participants were also asked if they believed the feedback and if they had any plans to change their drinking. Mediation analyses were implemented, exploring whether surprise, worry, or belief in the accuracy of feedback mediated severity of discrepancy's impact on plan for change.

Results: Among this sample of adult drinkers, severity of discrepancy did not predict plan for change, and personal dissonance did not mediate severity of discrepancy. Severity of discrepancy was mediated by belief in the accuracy of feedback. In addition, viewing one's drinking as a problem prior to feedback and post-NF worry both predicted plan for change independently. 
53 Conclusions: Results revealed that NF may not work to create personal dissonance through

54 discrepancy, but belief in the accuracy of feedback may be important. It appears the more one

55 believes the feedback, the more one makes a plan for change, suggesting practitioners should be

56 mindful of how information within feedback is presented. Findings also indicate NF may work

57 by validating a preexisting perception that drinking is a problem instead of creating concern

58 related to discrepancy where none existed. Limitations regarding generalizability are discussed. 
60

61

62

\section{Introduction}

Hazardous and/or harmful use of alcohol, such as drinking to excess or in the context of comorbid medical or mental health conditions, is a public health problem that causes economic and social burden across the globe ${ }^{1}$. In the United States in 2013, just under a quarter $(24.7 \%)$ of adults aged 18 and older reported binge drinking (consuming five or more drinks on one occasion), and 6.8\% reported heavy drinking (drinking beyond the recommended guidelines for safe alcohol use given by the National Institute for Alcohol Abuse and Alcoholism²) in the past month ${ }^{3}$. Many hazardous drinkers will be at risk for serious health consequences but do not perceive their drinking as requiring formal treatment ${ }^{4-6}$. Due to the prevalence rates of hazardous drinking, the avoidance of formal treatment, and the recent call for expanded prevention services within health care reform ${ }^{7}$, there is an increasing need for effective brief interventions that address harmful alcohol use at both the individual and population levels ${ }^{8,9}$ outside of specialty health care settings.

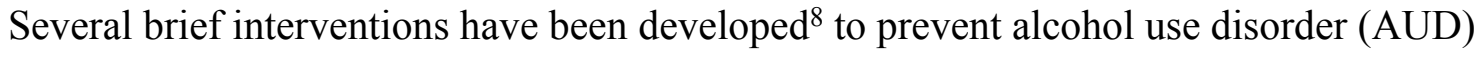
and to reduce alcohol related consequences. Brief interventions tend to be less than an hour long, or sometimes as short as a few minutes, and are usually implemented by a non-specialist, such as a primary care physician, or, in some cases, on a computer. One such brief intervention is normative feedback (NF), a widely used intervention for prevention of and early intervention for hazardous alcohol use ${ }^{10}$. During NF, individuals are assessed and then provided with information about how their drinking patterns compare to drinking norms of their peers of the same gender and age. The purpose of NF is to provide a drinker with a tool for understanding their own, potentially excessive, drinking and ultimately motivate them to reduce their drinking to safe levels. 

example, NF can be implemented as a standalone intervention; as part of more detailed feedback that includes information on health consequences, referred to as personalized feedback ${ }^{11}$; or alongside or within another therapeutic intervention, such as Motivational Enhancement Therapy $(\mathrm{MET})^{11,12}$. NF is often provided in community-based settings, such as primary care ${ }^{13,14}$, via multiple modalities (e.g., web-based, mailed, in-person) ${ }^{11,15-18}$. Given its relative simplicity, it is somewhat surprising that NF demonstrates consistent effectiveness in helping individuals reduce drinking at statistically significant levels across all modalities, contexts (e.g., within therapy or alone), and structures (e.g., a single item or detailed feedback about multiple health indicators $)^{4,9,11,12,15,17,19-27}$.

It is important to note that the preponderance of the above described research on NF has primarily focused on college students and/or their non-college same-age counterparts ${ }^{11,28,29}$. This is in part because of the well documented prevalence of high risk drinking on college campuses and among individuals at this stage of late adolescence ${ }^{3}$. Only a few studies have examined the impact of NF among adult drinkers beyond the college age years (e.g., 4,10,13,17,18,23,27). Within those few studies, NF again demonstrates preliminary effectiveness among adults at a spectrum of ages; however, this effectiveness is less well established, especially long term ${ }^{27}$, than for college-aged individuals. While NF is considered an effective intervention, particularly for individuals in late adolescence, it can potentially be improved to better facilitate both individual and population health $^{8}$. While some studies demonstrate impressive effect sizes of NF, most studies demonstrate small effect sizes $9,17,25$. There is also evidence that NF does not impact drinking outcomes as 
105 106

much as has been previously suggested ${ }^{30,31}$, particularly outside the context of primary care or for more than six months ${ }^{27}$.

One way to improve an intervention is to understand its mechanisms of action. If one identifies and understands how an intervention works, one can hone the intervention--improving its efficacy and cost-effectiveness. Although some studies have examined the mechanisms of action of personalized feedback ${ }^{8}$ and $\mathrm{NF}^{32}$, mechanisms remain relatively unexplored ${ }^{31}$. Proposed mechanisms of action of NF. Two potential mechanisms of action of NF explored in the literature are 1) discrepancy, including both external discrepancy ${ }^{33}$, the difference between one's perceived norm and the actual norm, and internal discrepancy ${ }^{33}$, the dissonance between an individual's drinking and an internal standard of comparison, such as one's "ideal" drinking behavior; and 2) perceived accuracy of feedback.

Discrepancy has been explored in a number of ways due to its different forms and definitions. Social Norms Theory ${ }^{34}$ is used to explain the effect of NF- such that once the perception of how much others drink is reduced, then the individual's drinking will be reduced in kind. Some studies support this theory, demonstrating change in perceived norms as a significant mediator of NF in reducing drinking among college students ${ }^{22,32,35-37}$. Other studies show that while change in perceived norms relates to intentions to change alcohol use among college students, it does not necessarily lead to actual reduced drinking ${ }^{33,38,39}$. Notably, young adults may react to discrepancy differently from older adults due to their particular life stage ${ }^{40}$. For example, a heightened need for peer approval during late adolescence may cause a more potent effect of $\mathrm{NF}^{23}$. As such, mechanisms of action of NF may differ across age groups. Within the context of MET ${ }^{41}$, NF is thought to develop internal, self-ideal discrepancy ${ }^{39,42}$. In MET, a heightened awareness of discrepancy between one's image of oneself 
128 and one's actual behavior is thought to create conflict for the individual, resulting in discomfort

129 with the status quo ${ }^{43}$ and thus enhancing motivation to change. This conflict is described many

130 ways — including affectively ${ }^{43}$ — such as fear, worry, or a neutral uneasiness with the difference

131 between perception and behavior. This conflict is conceptualized here as an affective response to

132 discrepancy and is termed personal dissonance. Only two studies thus far directly examined the

133 affective response related to NF and drinking with any population ${ }^{23,43}$. Results of one study

134 demonstrated that among adult problem drinking men-who-have-sex-with-men (MSM), NF

135 within MET elicited concern and worry, but these responses were not related to reduction in

136 post-NF drinking ${ }^{23}$. The second study $^{43}$ also found no relationship between developed affective

137 dissonance and drinking outcomes among heavy drinking college students. Given that only one

138 study has examined this process among an adult population beyond college age and only in the

139 context of MET, further research is needed to explore affective responses to NF and their role in

140 behavior change among adults, at a range of ages.

141 Perceived accuracy of NF (e.g., belief that norms used for drinking comparisons and the

142 results of the evaluation are true or valid) may also mediate NF's efficacy. Decision dilemma

143 theory is a behavioral economic theory that posits that poor decision making (e.g., continued

144 hazardous drinking) results from receiving "equivocal" feedback ${ }^{44,45}$ or "feedback for which

145 multiple (positive or negative) interpretations can be constructed" 45 . An individual who perceives

$146 \mathrm{NF}$ as highly equivocal might view the information as inaccurate or not applicable to their

147 personal situation. As a result, the individual would dismiss the information, nullifying the

148 effects of NF. In their study of adult drug users who received NF, Amrhein and colleagues ${ }^{46}$

149 noted a subgroup of participants who disagreed with the feedback they received (thus viewed it

150 as "highly equivocal"). This subgroup demonstrated more stable or increased drug use post-NF. 
151 Were this the mechanism of action of NF, it would be important to modify NF to present

152 information in a "believable way" to maximize its effect. A study of 90 adult problem drinking

153 MSM who received NF specifically tested this and found no support for DDT as an explanatory

154 mechanism for $\mathrm{NF}^{23}$. A primary problem with this study was that perceived accuracy of feedback

155 was measured by a third party observer. By viewing video-taped psychotherapy sessions in

156 which NF was provided, observers coded statements clients made in session to measure the

157 client's perceived accuracy of the feedback (e.g., "Your data is wrong."). In-session statements

158 made about this perception were not explicitly solicited by therapists and thus were made

159 inconsistently across participants. Participants were also not directly asked whether they believed

160 the feedback outside of session.

161 The current study aimed to expand the limited knowledge about the potential mechanisms

162 of action within NF, specifically affective responses to NF and belief in the accuracy of the

163 feedback, by implementing a feasibility and exploratory pilot study of NF on adult drinkers.

164 Given that many individuals with varied drinking patterns are receiving feedback via online

165 mechanisms, such as alcoholscreening.org, we explored whether it was possible to gather data

166 about personal reactions to feedback and test the mechanisms of action of web-based NF with a

167 heterogeneous sample of drinkers. In this feasibility study, personal dissonance, as defined by

168 level of surprise and worry, and belief in the accuracy of feedback were tested as mediators of

169 severity of discrepancy (the magnitude of the difference between one's drinking and peer norms)

170 on a drinker's plan to reduce drinking. It was hypothesized that both personal dissonance and

171 belief in feedback would emerge as mediators of severity of discrepancy predicting planning for

172 behavior change.

\section{Method}




\section{Recruitment}

Participants were recruited through Amazon Mechanical Turk (MTurk), an online labor market. MTurk is a online platform through which "workers" are contracted to complete tasks,

177 called "human intelligence tasks" (HITs), such as beta testing software or providing consumer opinions. For completing a HIT, workers are compensated by the publisher of the task, called

179 "requesters". Compensation is generally low-often times below $\$ 1$-and commensurate with 180 the task intensity. Increasingly, MTurk is being used for social sciences research with

181 comparable results to more traditional sampling methods, when validity checks are included in 182 the design ${ }^{47,48}$.

\section{Eligibility}

184 Participants were workers registered on MTurk. To participate in this HIT, they had to 185 have received a HIT approval rating of $95 \%$ out of at least 500 completed HITs by other requesters. This limited the sample to workers deemed acceptable by other requesters and with 187 adequate literacy in computer and internet use - thus providing increased assurance that participants could optimally understand this computer based intervention. Eligibility was limited to those residing in the United States.

190

192

\section{Procedures}

Eligible workers viewed the advertisement for the HIT Answer questions about a health

behavior and receive feedback. Within the HIT, participants were directed to a link to an external web-based survey, hosted by SurveyMonkey.com. The initial page of the web-based survey provided information about the study, and only those who consented to participate clicked through to the actual survey. Within the survey, participants answered the question: "do you drink alcohol?" Participants who answered no $(\mathrm{N}=38)$ were directed to a survey related to 
197 exercise and were excluded from this analysis. Those who responded yes were directed to a 198 survey about alcohol, which included NF about their own use (described further below). At the end of the survey, participants were provided with the reference information for the data used in

200 the feedback. The survey took about 6 minutes to complete. Once completed, participants were

201 provided with a code for their MTurk account, signaling the requester to review the responses

202 and approve compensation. Participants were compensated 50 cents for survey participation, an 203 average rate of compensation for comparable tasks. Workers were barred from retaking the 204 survey.

205

206

207

208

209

210

211

212

213

214

215

216

217

218

\section{Measures}

Demographics. Participants were asked their age and gender.

Perceptions of participants' drinking. Participants were asked a number of questions about their perception of their drinking prior to NF. On a scale of 1 (Not at all) to 8 (Extremely), participants were asked if the amount that they drank was excessive (excessive) and whether their current drinking is a problem for them or others (problem for others) in their lives. Finally, drinkers were asked "when you have not planned to drink, how much effort does it take for you not to drink alcohol when it is presented to you?" (effort). The effort question attempted to capture level of control over drinking.

Self-reported quantity and frequency of drinking. Participants were asked to report on their actual drinking through a series of questions based on the Form 90 QFV-30 ${ }^{49}$. Responses were used to provide NF, described below.

Personal dissonance. Immediately after feedback was provided, participants were asked to rate, again on a scale of 1 (Not at all) to 8 (Extremely), 1) how surprised are you by the 
219 feedback? (surprise) and 2) how much does the feedback worry you? (worry). These were used 220 as proxies for personal dissonance resulting from NF.

Expectation. Participants were asked "Is this feedback, ..?" and then provided with three

222

223

224

225

226

227

228

229

230

231

232

233

234

235

236

237

238

239

240

241

options: better than expected, as expected and worse than expected.

Newness of information. Participants were asked if the information provided in the NF was new. They could respond yes or no.

Plan to change. In the last section of the survey, participants were asked about their plan related to drinking for the next 30 days. This question was categorical and responses ranged from (0) no change in drinking to (3) quitting drinking. Participants were also asked to rate on a scale of 1 (Not at all) to 8 (Extremely) their commitment to and confidence in their ability to achieve their plan.

Belief in the accuracy of NF. To avoid biasing item responses earlier in the survey, the last question was "how accurate does the information you received about your drinking seem to you?" A 5-point Likert response scale included anchors -2 Not at all accurate to 2 Definitely accurate. Zero was "Not sure".

Severity of discrepancy. Participants were asked to rank themselves as to how much they drink compared to their same gendered peers in the form of a percentile rank. Severity of discrepancy was calculated as the difference between the participant's initial reported percentile rank and actual percentile rank (calculated based on reported standard drinks per week)--a measure similar to those used in other studies ${ }^{22,50}$. Higher numbers indicated greater discrepancy. Positive severity of discrepancy indicated that participants drank more compared to their peers than they estimated.

\section{Normative Feedback}


243 Health $^{51}$. Based on participants' reported average number of standard drinks per week, they were

244 directed to one of 18 different possible statements of feedback, each with a unique percentile

245 rank based on gender and quantity of standard drinks per week. Feedback contained two

246 sentences; for example: "You reported drinking 1 standard drink per week. You drink more than

$24727 \%$ of men." While we asked about binge drinking, binge drinking was not a factor in

248 determining feedback, nor were participants educated about binge drinking.

249 Analytic Plan

Descriptive analyses were performed to evaluate overall participant perceptions of health

251

252

253

254

255

256

257

258

259

260

261

262

263

behaviors, their reactions to NF, and their plans for change. Correlations between the perceptions of drinking pre-NF and post-NF reactions were calculated. Mediation analyses were performed using the PROCESS procedure ${ }^{52}$ in SPSS 22.0 (IBM, 2013). Mediation was formally tested using the product of coefficients and bias-corrected bootstrap confidence intervals ${ }^{52}$, with 10,000 samples specified. Mediators were first tested independently, and effect sizes of the indirect effects were calculated using Preacher and Kelley's kappa-squared ${ }^{53}$, where appropriate. In this context, Kappa-squared provides information about the size of the indirect effect relative to its maximum possible value, given the natural constraints imposed by variances and correlations between variables ${ }^{52}$. If more than one independent mediator was significant, then multiple mediation was tested. Procedures were reviewed and granted approval by the Institutional Review Board at the New York State Psychiatric Institute.

\section{Results}

\section{Sample description}


male.

266

267

268

269

270

271

272

273

274

275

276

277

278

\section{Descriptives}

Table 1 shows the reported drinking levels for the sample. Mean number of standard drinks per week for the entire sample was $8.1(\mathrm{SD}=13.1)$, with a range from 1 standard drink to as high as 105 for men and 60 to women. While the majority of participants were light to moderate drinkers, many participants reported drinking all of their drinks on only one or two days.

Based only on number of standard drinks per week, $14.9 \%$ reported drinking beyond safety guidelines set by the National Institute of Alcohol Abuse and Alcoholism for drinks per week among both men ( $>14$ standard drinks per week) and women ( $>7$ standard drinks per week).

Perceptions of drinking pre-NF. There were no gender differences on any of the perception variables. Table 1 demonstrates how participants viewed their drinking. Overall, drinkers did not view their drinking as excessive, a problem, or a problem for others. Refusing a drink required little effort. Drinkers also tended to largely underestimate their drinking compared to US adults, as demonstrated by a high mean severity of discrepancy score.

\section{[Insert Table 1 here.]}

Reactions to NF. Reactions to NF are shown in Table 2. While participants were generally surprised by feedback, they were not particularly worried by it. Only $12.6 \%$ of participants viewed feedback as better than expected or as expected. A preponderance of drinkers $(87.4 \%)$ reported feedback was worse than expected. A majority of drinkers (88\%) reported this information was new. On average, drinkers reported that the feedback was somewhat inaccurate. 
287

288

289

290

291

292

293

294

295

296

297

298

299

300

301

302

303

304

305

306

307

308

309

[Insert Table 2 here.]

Plan, confidence, and commitment to change. Table 2 also shows the outcome of NF. Among this sample of drinkers, $26.4 \%$ planned to quit or reduce their drinking. Confidence and commitment to change drinking were high.

\section{Correlations between Perception of Drinking Pre-NF and Post-NF Reactions}

Correlation coefficients between pre-NF perceptions of drinking and the mediators were generated (See Table 3). Drinking is a problem was significantly negatively correlated with surprise and significantly positively correlated with worry, belief in the accuracy offeedback, and effort. Drinking is a problem was also significantly negatively correlated with severity of discrepancy. Findings suggest when an individual viewed his or her alcohol use as problematic, there was higher accuracy in his or her estimate of how his or her drinking compared to the norm. As a result, feedback was believable and not a surprise.

[Insert Table 3 here.]

Drinking is a problem prior to NF was significantly and strongly correlated with plan for change (Table 3), such that the greater the participant ranked drinking is a problem, the more ambitious the plan for reduced drinking.

\section{Total Effect of Severity of Discrepancy on Plan for Change}

There was no total effect of severity of discrepancy on plan for change $(b=.003, S E=$ $.01, p=.79)$

\section{Mediation Models for Personal Dissonance (Worry and Surprise)}

Table 4 displays the relationships of the mediation models. There were no significant indirect effects for either worry or surprise on plan for change. For this sample, personal dissonance did not mediate the effect of severity of discrepancy on making a plan for change. 
[Insert Table 4 here.]

\section{Mediation Models for Belief in the Accuracy of NF}

Table 4 also shows the results from the mediation model for belief in the accuracy of

313 feedback. Severity of discrepancy was significantly negatively associated with belief in the

314 accuracy of feedback. There was a significant indirect effect for belief in the accuracy of

315 feedback $(.004,95 \%$ CI $[.001, .01])$. The kappa-squared statistic revealed that the indirect effect

316 is $11.6 \%$ of its maximum possible value $\left(\mathrm{K}^{2}=.116, S E=.06,95 \% \mathrm{CI}[.03, .25]\right)$.

\section{Post Hoc Analyses}

Since this was a sample of mostly light to moderate drinkers, rather than predominantly

hazardous drinkers, it was surprising to discover such a large proportion of them $(26.4 \%)$

reported planning at least some change in their drinking. Due to the absence of a relationship linear regression.

Pre-NF drinking is a problem significantly predicted plan to change $(b=.24, S E=.05, p$

$<.001)$. When post-NF worry was entered as a second step in the model, both remained significant (drinking is a problem: $b=.24, S E=.05, p<.001 ;$ worry: $b=.24, S E=.05, p<.001$; adjusted $\left.R^{2}=.38 ; F(2,84)=26.8, p<.001\right)$, suggesting that both baseline perception that drinking is a problem and post-NF worry predict plan for change. Tolerance statistics demonstrated no multicollinear effect.

\section{Discussion}


This is the first study to test both personal dissonance and perceived accuracy of feedback

334 as mechanisms of action within NF among adult drinkers outside the context of MET.

335 Hypotheses that severity of discrepancy would be mediated by personal dissonance and belief in

336 the accuracy of feedback were only partially supported. While the participants in this study were

337 primarily light to moderate drinkers, a number of them greatly under-estimated their level of

338 drinking compared to their same gendered peers, demonstrating a wide range of discrepancy.

339 Still, severity of discrepancy was not associated with plan to change, which is inconsistent with

340 previous studies on college students ${ }^{22,50}$. Interestingly, findings were consistent with previous

341 research on a sample of hazardous drinkers from the general population ${ }^{10}$. This recent study

342 found that norm comparisons, provided in the context of more detailed personalized feedback,

343 did not appear to be the active mechanism for change among a sample of adult hazardous

344 drinkers ${ }^{10}$. It is possible that peer comparisons work especially well for individuals in their late

345 adolescence and emerging adulthood because it leverages their particular need for conforming

346 with peers' behaviors. Given the lack of association between severity of discrepancy and plan for

347 change, it also suggests that among a sample of mostly light to moderate drinkers, factual

348 normative discrepancy does not act alone to instigate thoughts about change.

While there were few heavy drinkers in this sample, lack of findings regarding personal

350 dissonance was consistent with previous studies on affective responses ${ }^{23,43}$ of heavy drinkers.

351 According to evidence thus far, it appears that affective responses are not associated with

352 outcomes of feedback among both college-age and an age-inclusive sample of adults with a

353 spectrum of drinking patterns.

Perceived accuracy of feedback was demonstrated to be a potential mediator of severity

of discrepancy, providing initial support for decision dilemma theory. Results suggest that the 
356 more an individual is aware of how much he or she drinks compared to others, the more likely he

357 or she will perceive the feedback to be accurate and thus more likely to set a plan for change.

358 Among this sample, this was true regardless of intensity of drinking. It is important to note that

359 the mediation effect of belief in the accuracy of $N F$ was quite small-only $11.6 \%$ of its possible

360 maximum value. More research is needed to further understand this potential mediator of

361 change.

362

Post hoc analyses support the possibility that feedback for drinkers may not operate to

363

364

365

366

367

368

369

370

371

372

373

374

375

376

377

378

create concern about or awareness of excessive drinking where none existed. Instead, it seems to potentially capitalize on a pre-existing perception that drinking is a problem, particularly among

those who may drink more heavily. Some studies demonstrate NF works best for those with the most severe drinking patterns ${ }^{54,55}$. It is therefore possible that feedback works within a framework of consonance rather than dissonance. This may explain why a "boomerang effect", an effect in which light drinkers who receive feedback about their drinking increase instead of decrease their drinking ${ }^{56}$, has yet to be found within the empirical literature. Furthermore, given the correlational relationships, perceiving alcohol is a problem may also indicate an awareness of one's drinking and already how it compares to existing norms.

Given that NF is one of the most widely used interventions to prevent and/or reduce hazardous drinking, the above findings have potentially important implications for application to clinical practice. When implementing NF to an adult sample, providers should be aware of both the existing concerns and perceptions a drinker may have, as well as how believable the information imparted is for the recipient. Inquiring about a patient's drinking and their perception of its role in his or her life a priori may help providers navigate providing feedback in an optimal way. For example, there may be little need to offer much additional feedback when 
379 one already perceives their drinking as problematic. Instead, offering an opportunity to explore

380 goal planning and/or treatment may be the best use of a clinician's time. For those who do not

381 perceive their drinking as problematic, feedback interventions may be best improved by

382 increasing the credibility of the feedback for the individual. By being selective in how one

383 provides feedback, providers may enhance its effectiveness.

\section{$384 \quad$ Limitations}

There are several limitations to this study, and findings should be interpreted in the context of an exploratory pilot study. While this study demonstrated the feasibility of measuring

387

388

389

390

391

392

393

394

395

396

397

398

399

400

401 affective responses and belief in the accuracy of feedback post-NF, generalizability is extremely limited. These participants were recruited from an online labor market, not the general population. The measurement of belief in the accuracy of feedback was simple in that it was a single item, self-report measure; however, it had face validity in that it captured the participant's perception rather than behavior, which was the specific construct of interest. A major limitation of this study is that there were few individuals who were heavy or hazardous drinkers, in terms of quantity of standard drinks per week, and thus results cannot be generalized to hazardous drinkers. More research on understanding this relationship with hazardous drinkers is needed; however, the greater variability in the sample allowed us to understand feedback in a heterogeneous drinking sample, such as those found in primary care or other opportunistic settings, in which NF is often used preventively. other studies have used a similar outcome, it is not equivalent to behavior. Finally, it is not known whether personal dissonance is experienced only in a proximal fashion. For example, a 
402 person who receives feedback may not initially experience worry but may find his or her concern

403 grows over a period of time. This potential sleeper effect of NF could in turn potentially affect

404 the timing of making a plan for change. Longitudinal research is needed to better understand the

405 timing of potential mediators and subsequent behavioral outcomes.

406 Future Research

407

Future research could include experimental manipulation with better operationalization of

408

409

410

411

412

413

414

415

416

417

418

419

420

421

422

423

424 normative feedback, self-ideal feedback and emotional responses in order to unpack the active ingredients of NF. Expanding the population upon which NF is tested to heavy drinkers and other undesirable health behaviors will be crucial to confirming these preliminary findings. Additionally, both actual behavioral outcomes and longer term, prospective perceptions of feedback must be explored to understand the mechanisms of maintenance of behavior change.

\section{Conclusions}

Despite the limitations, findings have the potential for immediate clinical application, and thus implications for individual and population level health. For individuals with a skewed perception of how much they drink compared to their peers, helping them to understand NF in context and making it believable (e.g., supported by empirical data) may be critical for maximum effectiveness of NF among adults. In addition, exploring with drinkers how much they are already perceive their drinking to be a problem may help to potentiate the effects of the simplest form of NF. These are relatively minor and feasible changes to NF that may in fact help to increase its efficacy rate, potentially helping to reduce the prevalence rate of hazardous alcohol use or prevent the development of AUD across a wide group of drinkers. 
Table 1. Pre-feedback descriptives of participants

$M(\mathrm{SD})$ or $\%$

\section{Males $(\mathbf{N}=56)$}

Alcohol Use is Excessive ${ }^{a}$

$1.9(1.5)$

Drinking is a Problem ${ }^{\text {a }}$

$1.6(1.3)$

My Drinking is a Problem for Others ${ }^{\mathrm{a}}$

$1.9(1.6)$

Effort Needed to Refuse a Drink ${ }^{\mathrm{a}}$

$2.6(1.7)$

Number of Days Drink per Week

Drinks per Drinking Dayb

$2.2(2.0)$

Binge Drank in the Last 30 Days $^{\mathrm{c}}$

$2.9(2.5)$

$62 \%$

Mean Standard Drinks per Week ${ }^{\mathrm{b}}$

$8.2(14.2)$

\section{Females $(\mathbf{N}=31)$}

Alcohol Use is Excessive ${ }^{a}$

Drinking is a Problem ${ }^{a}$

My Drinking is a Problem for Others ${ }^{\mathrm{a}}$

$1.8(1.5)$

Effort Needed to Refuse a Drink ${ }^{\mathrm{a}}$

$2.8(2.4)$

Number of Days Drink per Week

Drinks per Drinking Dayb

$1.8(1.5)$

Binge Drank in the Last 30 Days $^{\mathrm{c}}$

$2.8(2.4)$

$60 \%$

Mean Standard Drinks per Week ${ }^{b}$

$7.9(12.3)$

Severity of Discrepancy $^{d}(\mathbf{N}=87)$

$42.9(21.7)$ 
Table 2. Post Feedback Descriptives: Mediators and Outcome

$(\mathrm{N}=87)$

$M(\mathrm{SD})$ or $\%$

Personal Dissonance Variables

Surprise

$6.1(2.2)$

Worry

Expectation. This information was:

Better than Expected

2.3

As Expected

10.3

Worse than Expected

87.4

Yes, this information was new.

Belief the Feedback was Accurate ${ }^{a}$

Planning for Change

I have no plan to change my drinking.

I plan to cut back on my drinking a little bit.

I plan to greatly cut back my drinking.

9.2

I plan to quit drinking.

Confidence to Change Drinking $(n=23)$

Commitment to Change Drinking $(\mathrm{n}=23)$

$5.8(1.7)$

${ }^{\text {a }}$ Range was from -2 (Not at all accurate) to 0 (Not sure) to 2 (Definitely accurate). 


\begin{tabular}{|c|c|c|c|c|c|c|c|c|}
\hline & 1 & 2 & 3 & 4 & 5 & 6 & 7 & 8 \\
\hline & \multicolumn{8}{|c|}{$\underline{\text { Pre-NF Variables }}$} \\
\hline 1. Drinking is excessive & -- & & & & & & & \\
\hline 2. Drinking is a problem & $.92 * * *$ & -- & & & & & & \\
\hline 3. Drinking is a problem for others & $.47 * * *$ & $.51 * * *$ & -- & & & & & \\
\hline 4. Effort & $.49 * * *$ & $.46^{* * *}$ & $.33 * *$ & -- & & & & \\
\hline \multirow[t]{2}{*}{ 5. Severity of discrepancy } & $-.26^{*}$ & $-.29 * *$ & -.18 & -.16 & -- & & & \\
\hline & \multicolumn{8}{|c|}{$\underline{\text { Post-NF Variables }}$} \\
\hline 6. Surprise & -.15 & $-.39 * * *$ & -.15 & -.14 & $.55 * * *$ & -- & & \\
\hline 7. Worry & $.28 * *$ & $.47 * * *$ & -.15 & $.28 * *$ & .03 & -.07 & -- & \\
\hline 8. Belief in the accuracy of NF & .20 & $.34 * *$ & -.15 & -.20 & $-.37 * * *$ & $-.60 * * *$ & .18 & -- \\
\hline 9. Plan for change ${ }^{a}$ & $.42 * * *$ & $.47 * * *$ & $.26^{*}$ & .21 & -.10 & -.21 & $.58 * * *$ & $.32 * *$ \\
\hline
\end{tabular}

$* p<.05, * * p<.01, * * * \mathrm{p}<.001$.

${ }^{a}$ Ranged from 0 to 3 , with 0 indicating no plan for change and 3 indicating quitting drinking.

Note: $\mathrm{NF}=$ normative feedback 
Table 4. Mediation models for worry, surprise, and perceived accuracy of feedback predicting plan to change drinking

\begin{tabular}{|c|c|c|c|c|c|c|c|c|}
\hline \multirow[b]{3}{*}{ Model 1} & \multicolumn{8}{|c|}{ Outcome Variable } \\
\hline & \multicolumn{4}{|c|}{ Mediator } & \multicolumn{4}{|c|}{ Plan to Change Drinking } \\
\hline & & Coeff & $S E$ & $\boldsymbol{P}$ & & Coeff & $S E$ & $\boldsymbol{P}$ \\
\hline X (Severity of Discrepancy) & $a$ & .003 & .01 & .79 & $c^{\prime}$ & .004 & .003 & .19 \\
\hline \multirow[t]{2}{*}{ M (Worry) } & & -- & -- & -- & $b$ & .21 & .03 & $<.001$ \\
\hline & & \multicolumn{3}{|c|}{$\begin{array}{c}R^{2}=.001 \\
F(1,85)=0.07, p=.79\end{array}$} & & \multicolumn{3}{|c|}{$\begin{array}{c}R^{2}=0.35 \\
F(2,84)=22.8, p<.001\end{array}$} \\
\hline Model 2 & & Coeff & $S E$ & $\boldsymbol{P}$ & & Coeff & $S E$ & $\boldsymbol{P}$ \\
\hline X (Severity of Discrepancy) & $a$ & .06 & .01 & $<.001$ & $c^{\prime}$ & -.001 & .01 & .82 \\
\hline \multirow[t]{2}{*}{ M (Surprise) } & & -- & -- & -- & $b$ & .08 & .05 & .09 \\
\hline & & \multicolumn{3}{|c|}{$\begin{array}{c}R^{2}=.30 \\
F(1,84)=35.9, p<.001\end{array}$} & & \multicolumn{3}{|c|}{$\begin{array}{c}R^{2}=0.04 \\
F(2,83)=1.9, p=.16\end{array}$} \\
\hline Model 3 & & Coeff & $S E$ & $\boldsymbol{P}$ & & Coeff & $S E$ & $\boldsymbol{P}$ \\
\hline X (Severity of Discrepancy) & $a$ & -.02 & .01 & $<.001$ & $c^{\prime}$ & -.001 & .004 & .84 \\
\hline \multirow[t]{2}{*}{ M (Perceived Accuracy) } & & -- & -- & -- & $b$ & -.22 & .07 & $<.01$ \\
\hline & & \multicolumn{3}{|c|}{$\begin{array}{c}R^{2}=.13 \\
F(1,85)=13.0, p<.001\end{array}$} & & \multicolumn{3}{|c|}{$\begin{array}{c}R^{2}=0.11 \\
F(2,84)=4.9, p<.001\end{array}$} \\
\hline
\end{tabular}




\section{References}

431 1. World Health Organization. Global status report on alcohol and health 2014. Geneva, $432 \quad$ Switzerland: World Health Organization;2014.

433 2. National Institute on Alcohol Abuse and Alcoholism. Rethinking drinking. Bethesda, 434 MD: Author;2013.

435 3. Substance Abuse and Mental Health Services Administration. Results from the 2013 436 National Survey on Drug Use and Health: Summary of National Findings, NSDUH Series H-48, HHS Publication No. (SMA) 14-4863. Rockville, MD: Substance Abuse and Mental Health Services Administration;2014.

4. Cunningham J, Wild TC, Bondy SJ, Lin E. Impact of normative feedback on problem drinkers: A small-area population study. Journal of Studies in Alcohol. 2001;62:228-233.

5. Hester R, Delaney HD. Behavioral self control program for windows: Results of a controlled clinical trial. Journal of Consulting and Clinical Psychology. 1997;65(4):686-

6. Rosenberg H. Prediction of controlled drinking by alcoholics and problem drinkers. Psychological Bulletin. 1993;113(1):129-139.

7. Arvantes J. Health care reform law will increase demand for preventive services, say experts AAFP News. 2010. http://www.aafp.org/news/government-

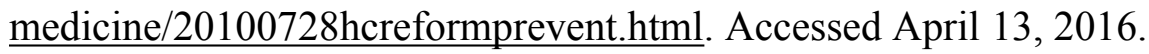

8. McCambridge J, Cunningham JA. The early history of ideas on brief interventions for 450 alcohol. Addiction. 2014;109(4):538-546. PMC3992901 
4519 9. Dotson KB, Dunn ME, Bowers CA. Stand-alone personalized normative feedback for 452 college student drinkers: A meta-analytic review, 2004 to 2014. PLoS One.

453 2015;10(10):e0139518. PMC4598082

454 10. Cunningham J, Murphy M, Hendershot CS. Treatment dismantling pilot study to identify

455 the active ingredients in personalized feedback interventions for hazardous alcohol use: randomized controlled trial. Addict Sci Clin Pract. 2015;10:1. PMC4288561

457 11. Walters ST, Neighbors C. Feedback interventions for college alcohol misuse: What, why, and for whom? Addict Behav. 2005;30:1168-1182.

459 12. Murphy JG, Benson TA, Vuchinich RE, Deskins MM, Eakin D, Flood AM, McDevittMurphy ME, Torrealday O. A comparison of personalized feedback for college student drinkers delivered with and without a motivational interview. Journal of Studies in

13. Maisto SA, Conigliaro J, McNeil M, Kraemer K, Conigliaro RL, Kelley ME. Effects of two types of brief intervention and readiness to change on alcohol use in hazardous drinkers. J Stud Alcohol. 2001;62(5):605-614.

14. Collins SE, Carey KB, Sliwinski MJ. Mailed personalized normative feedback as a brief intervention for at-risk college drinkers. J Stud Alcohol. 2002;63:559-567.

15. White HR. Reduction of alcohol-related harm on United States college campuses: The use of personal feedback interventions. International Journal of Drug Policy. 2006;17:310-319.

16. Walters ST, Woodhall WG. Mailed feedback reduces consumption among moderate drinkers who are employed. Prevention Science. 2003;4(4):287-294. 
473 17. Cunningham JA, Neighbors C, Wild C, Humphreys K. Ultra-brief intervention for

474

475

476

477

478

479

480

481

482

483

484

485

486

487

488

489

490

491

492

493

494

495

problem drinkers: Results from a randomized controlled trial. PLoS One.

2012;7(10):e48003.

18. Cunningham JA, Wild TC, Cordingley J, Van Mierlo T, Humphreys K. A randomized controlled trial of an internet-based intervention for alcohol abusers. Addiction. 2009;104(12):2023-2032.

19. Miller WR, Benefield R, Tonigan JS. Enhancing motivation for change in problem drinking: a controlled comparison of two therapist styles. Journal of Consulting and Clinical Psychology. 1993;61(3):455-461.

20. White HR, Mun EY, Pugh L, Morgan T. Long-term effects of brief substance use interventions for mandated college students: Sleeper effects of an in-person personal feedback intervention. Alcoholism: Clinical and Experimental Research. 2007;31(8):1380-1391.

21. Doumas DM, Hannah E. Preventing high-risk drinking in youth in the workplace: A webbased normative feedback program. Journal of Substance Abuse Treatment. 2008;34:263271.

22. Walters ST, Vader AM, Harris TR, Field C, Jouriles EN. Dismantling Motivational Interviewing and feedback for college drinkers: A randomized clinical trial. Journal of Consulting and Clinical Psychology. 2009;77(1):64-77.

23. Kuerbis A, Schaumberg K, Davis CM, Hail L, Morgenstern J. Unpacking personalized feedback: An exploratory study of the impact of its components and the reactions it elicits among problem drinking men who have sex with men. Substance Use and Misuse. 2014;49(4):383-394. 
496 24. Riper H, van Straten A, Keuken M, Smit F, Schippers G, Cuijpers P. Curbing problem

497 drinking with personalized-feedback interventions: A meta-analysis. Am J Prev Med. $498 \quad 2009 ; 36(3): 247-255$.

499 25. Cadigan JM, Haeny AM, Martens MP, Weaver CC, Takamatsu SK, Arterberry BJ. Personalized drinking feedback: A meta-analysis of in-person versus computer-delivered interventions. J Consult Clin Psychol. 2015;83(2):430-437. PMC4380651

26. Zimmerman D, Fischhoff B. A corrective meta-analysis of personalized normative feedback. Dietrich College of Humanities and Social Sciences at Research Showcase@ CMU. 2014.

27. Cunningham J, Wild TC, Cordingley J, Van Mierlo T, Humphreys K. Twelve-month follow up results from a randomized controlled trial of a brief personalized feedback intervention for problem drinkers. Alcohol and Alcoholism. 2010;45:258-262.

28. Reid AE, Carey KB. Interventions to reduce college student drinking: State of the evidence for mechanisms of behavior change. Clin Psychol Rev. 2015;40:213-224. PMC4537835

29. Miller MB, Leffingwell T, Claborn K, Meier E, Walters S, Neighbors C. Personalized feedback interventions for college alcohol misuse: An update of Walters \& Neighbors (2005). Psychol Addict Behav. 2013;27(4):909-920.

514 30. Foxcroft DR, Moreira MT, Almeida Santimano NM, Smith LA. Social norms information for alcohol misuse in university and college students. Cochrane Database Syst Rev. 2015;1:CD006748. 
517 31. Gaume J, McCambridge J, Bertholet N, Daeppen JB. Mechanisms of action of brief

518 alcohol interventions remain largely unknown - a narrative review. Frontiers in

$519 \quad$ Psychiatry. 2014;5:108. PMC4143721

520 32. Neighbors C, Lewis MA, LaBrie J, DiBello AM, Young CM, Rinker DV, Litt D, Rodriguez LM, Knee CR, Hamor E, Jerabeck JM, Larimer ME. A multisite randomized trial of normative feedback for heavy drinking: Social comparison versus social comparison plus correction of normative misperceptions. J Consult Clin Psychol. 2016.

524 33. Neal DJ, Carey KB. Developing discrepancy within self-regulation theory: Use of personalized normative feedback and personal strivings with heavy-drinking college students. Addict Behav. 2004;29:281-297.

34. Perkins HW. The emergence and evolution of the social norms approach to substance abuse prevention. In: Perkins HW, ed. The social norms approach to preventing school and college age substance abuse: A handbook for educators, counselors and clinicians. San Francisco, CA: Jossey-Bass; 2003.

35. Borsari B, Carey KB. Effects of a brief motivational intervention with college student drinkers. Journal of Consulting and Clinical Psychology. 2000;68(4):728-733.

36. Neighbors CJ, Larimer ME, Lewis MA. Targeting misperceptions of descriptive drinking norms: Efficacy of a computer-delivered personalized normative feedback intervention. Journal of Consulting and Clinical Psychology. 2004;72(3):434-447.

37. Walters ST, Vader AM, Harris TR. A controlled trial of web-based feedback for heavy drinking college students. Prevention Science. 2007;8:83-88.

538 38. Larimer ME, Lee CM, Kilmer JR, Fabiano PM, Stark CB, Geisner IM, Mallett KA, 539 Lostutter TW, Cronce JM, Feeney M, Neighbors C. Personalized mailed feedback for 
college drinking prevention: A randomized clinical trial. Journal of Consulting and Clinical Psychology. 2007;75(2):285-293.

542 39. Murphy JG, Dennhardt AA, Skidmore JR, Martens MP, McDevitt-Murphy ME.

543 Computerized versus motivational interviewing alcohol interventions: Impact on

544 discrepancy, motivation, and drinking. Psychol Addict Behav. 2010;24(4):628-639.

545 40. Erikson EH. Identity: Youth and crisis. New York: Norton; 1968.

546 41. Miller WR, Zweben A, DiClemente CC, Rychtarik RG. Motivational Enhancement Therapy manual: A clinical research guide for therapists treating individuals with alcohol abuse and dependence. Rockville, MD: National Institute for Alcohol Abuse and Alcoholism;1999.

42. Miller WR, Rollnick S. Motivational interviewing: Preparing people for change. 2nd ed. New York: The Guilford Press; 2002.

43. McNally AM, Palfai TP, Kahler CW. Motivational intervenitons for heavy drinking college students: Examining the role of discrepancy-related psychological processes. Psychol Addict Behav. 2005;19(1):79-87.

555

556

557

558

559

560

561

562

44. Hantula DA, DeNicolis Bragger JL. The effects of feedback equivocality on escalation of commitment: An empirical investigation of decision dilemma theory. Journal of Applied Social Psychology. 1999;29(2):424-444.

45. Bowen MG. The escalation phenomenon reconsidered: Decision dilemmas or decision errors? Academy of Management Review. 1987;12:52-66.

46. Amrhein PC, Miller WR, Yahne CE, Palmer M, Fulcher L. Client commitment language during motivational interviewing predicts drug use outcomes. Journal of Consulting and Clinical Psychology. 2003;71(5):862-878. 
563 47. Mason W, Suri S. Conducting behavioral research on Amazon's Mechanical Turk.

564

565

566

567

568

569

570

571

572

573

574

575

576

577

578

579

580

581

582

583

584

585

Behavior Research Methods. 2012;44(1):1-23.

48. Muench F, van Stolk-Cooke K, Morgenstern J, Kuerbis A, Markle K. Understanding messaging preferences to inform development of mobile goal-directed behavioral interventions. Journal of Medical Internet Research. 2014;16(1):e14.

49. Miller WR, Del Boca FK. Measurement of drinking behavior using the Form 90 family of instruments. J Stud Alcohol. 1994;Suppl. 12:112-118.

50. Walters ST, Vader AM, Harris TR. A controlled trial of web-based feedback for heavy drinking college students. Prevention Science. 2007;8(1):83-88.

51. Substance Abuse and Mental Health Services Administration. Results from the 2009 National Survey on Drug Use and Health: Summary of national findings, NSDUH Series H-38A, HHS Publication No. (SMA) 10-4856. Rockville, MD: Substance Abuse and Mental Health Services Administration;2010.

52. Hayes AF. Introduction to mediation, moderation, and conditional process analysis: A regression based approach. New York: The Guilford Press; 2013.

53. Preacher KJ, Kelley K. Effect size measures for mediation models: quantitative strategies for communicating indirect effects. Psychol Methods. 2011;16(2):93-115.

54. Murphy JG, Duchnick JJ, Vuchinich RE, Davison JW, Karg RS, Olson AM, Smith AF, Coffey TT. Relative efficacy of a brief motivational intervention for college student drinkers. Psychol Addict Behav. 2001;15(4):373-379.

55. Doumas DM, Andersen LL. Reducing alcohol use in first-year university students: Evaluation of a web-based personalized feedback program. Journal of College Counseling. 2009;12(1):18-32. 
586 56. Prince MA, Reid A, Carey KB, Neighbors C. Effects of normative feedback for drinkers

587 who consume less than the norm: Dodging the boomerang. Psychol Addict Behav.

588 2014;28(2):538-544. PMC4169697

589 